\title{
Silicon Nanowire Immunosensor for Detection of 8-Hydroxy-2'-Deoxyguanosine Oxidative Stress Cancer Biomarker
}

\author{
D. J. Thomas* \\ Centre for NanoHealth, Singleton Hospital, Singleton Park, Swansea, SA2 8PP, U. K. \\ Z. Therani \\ Welsh Centre for Printing and Coating, College of Engineering, \\ Swansea University, Singleton Park, Swansea, SA2 8PP, U. K. \\ M. A. B. Mohd Azmi \\ University Kuala Lumpur, British Malaysian Institute, Gombak, Selangor, Malaysia \\ (Received 6 January 2014; Accepted 22 April 2014; Published 26 July 2014)
}

\begin{abstract}
A silicon nanowire-based immunosensor has been developed to determine the presence of 8-hydroxy-2'deoxyguanosine $(8-\mathrm{OHdG})$. This research demonstrates a process for the detection of 8-OHdG, which is an oxidative stress biomarker, in which elevated concentrations in urine and saliva can act as an indicator of the presence of acute leukaemia, colorectal, breast, lung and prostate cancers. The detection of elevated levels of this biomarker is critical in determining the presence of various mutagenesis and can be used to diagnose early stage cancer before initial symptoms appear. The surface functionalization process has involved the attachment of diazonium salt to the silicon nanowire surface with further stage which involves the reduction of $\mathrm{PhNO}_{2}$ to $\mathrm{PhNH}_{2}$. The antibody targeted against 8-OHdG was subsequently bound to the amine $\mathrm{NH}_{2}$ modified grafted layer of a $370 \mathrm{~nm}$ width nanowire. The immunosensor was evaluated through the use of current/voltage electrical probe monitoring in which a $2 \mu \mathrm{g} / \mathrm{ml}$ concentration of $8-\mathrm{OHdG}$ was detected. A further comparison with phosphate buffered saline solution added to the sensor was observed to be negligible. The antibody-functionalised SiNW sensor has been used to detect binding of the 8-OHdG biomarker to the SiNW surface within sixty-seconds of exposure, making this a rapid cancer biomarker test. [DOI: 10.1380/ejssnt.2014.349]
\end{abstract}

Keywords: Silicon nanowire; Immunosensor; Cancer biosensing; Biological functionalization; 8-hydroxydeoxyguanosine (8OHdG)

\section{INTRODUCTION}

Lung, breast and prostate cancers were the three leading causes of cancer-related death in the US between 2004 and 2007, claiming over 227,900 lives in 2007 alone. Treatments are more effective if detection is made early. A reliable nanowire-based immunosensor with a selected and calibrated sensitivity level can be used to rapidly diagnose cancers at the earliest stages [1]. Semiconductor nanowire-based field effect transistors (FET) allow the ultrasensitive electrical detection of biomolecular interactions [2]. Nanowire-based devices enable detection through the process of changes in electrical conductance across a nanowire [3-6]. This process is detected through a conductance change in response to variations in the electric field of the device [7-11]. The resulting effect is that there is an change in device conductance, which is dependent on conductance on induced gate voltage and corresponding charge at the gate electrode interface makes FETs capable of electrical-based sensing.

Nanowires composed of silicon can function as FET devices [9-11]. Binding of charged or polar biological or chemical species to the gate dielectric is comparable to applying a voltage using a gate electrode. An analyte binding to the surface of a nanowire can lead to depletion or accumulation of carriers through the entire cross section of the device. In the case of a $p$-type semiconductor, applying a negative gate voltage results in an accumula-

*Corresponding author: d.j.thomas@swansea.ac.uk tion of majority charge carrier positive holes and results in a corresponding increase in conductance.

A silicon nanowire (SiNW)-based immunosensor offers a means to detect the selective and abnormally high levels of a biomarker to indicate the presence of a disease and make an accurate early diagnosis. These exhibit a conductance change in response to variations in the charge or potential at the surface of the channel region [12]. It is this characteristic that makes FETs appropriate for electrically based sensing mechanism detection. This unique feature of semiconductor nanowires enables the ability for a high degree of sensitivity that opens up the prospect for interfaces with biological systems [13].

Thinner SiNWs are more influenced by surface charges which induce a depletion or accumulation region in the SiNW, resulting in a greater effect on the conductance/resistance of the SiNW sensor device. Consequently, many SiNW sensor fabrication processes used a tetra methyl ammonium hydroxide (TMAH) wet etchant nanowire thinning method to reduce the diameter of the nanowire [14-16]. The sensitivity and selectivity of nanowire-based FET detectors has subsequently been demonstrated in the context of protein detection. Patolsky and Lieber [3] have explored the sensitivity limits of nanowire-based FETs functionalized with monoclonal antibodies that were specific to cancer detection markers such as antigens linked to prostate cancer. Nanomaterials have critical high aspect ratio physical properties that have been proven by to be very specific in detecting cancer biomarkers [3].

The development of silicon nanowire sensor arrays for cancer biomarker detection has also been carried out by Wang et al. [6] and Zheng et al. [13] who attached mon- 


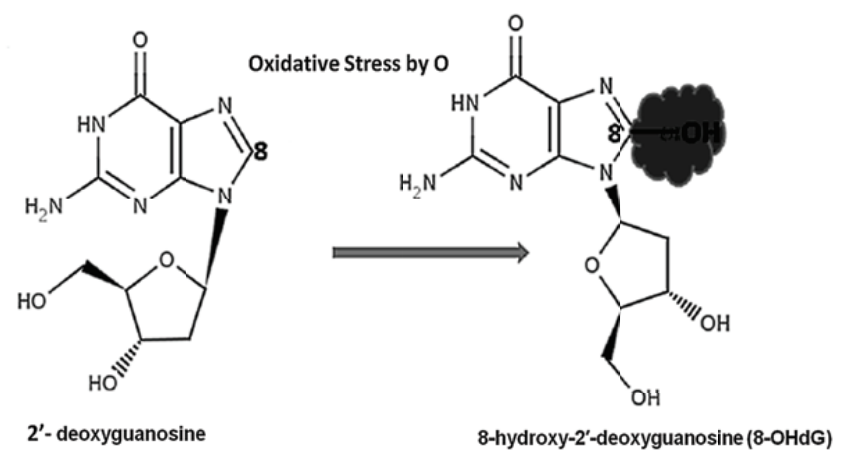

FIG. 1: Formation of 8-hydroxy-2'-deoxyguanosine (8-OHdG) from 2'-deoxyguanosine [22].

oclonal antibodies to the nanowire elements following device fabrication. Sensitivity limits for cancer biomarker protein detection using this new generation of silicon nanowire device arrays were again determined by measuring conductance changes as the solution concentration of prostate specific antigen (PSA). Zheng et al. [13] observed that direct, label-free detection of biomarkers can be achieved for concentrations down to $75 \mathrm{fg} / \mathrm{ml}$.

Methods for the covalent functionalization of SiNWs including amino termination using (3-aminopropyl) triethoxysilane (APTES) linking chemistry, which has previously been applied to detect DNA and peptide nucleic acid (PNA) attachment to SiNW in DNA/PNA biosensors [10, 17-19], and photochemical grafting using alkene derivatives [20].

8-hydroxy-2'-deoxyguanosine (8-OHdG) under examination during this research is a product of DNA radical damage and can be used as an indicator of damage to DNA [21]. As a result of cancer, the body is placed under stress which produces radical oxygen atoms. These attack the $\mathrm{C} 8$ position of 2'-deoxyguanosine and introduce a hydroxyl group at the $\mathrm{C} 8$ position to produce $8-\mathrm{OHdG}$ as Fig. 1 shows [22]. $8-\mathrm{OHdG}$ is thus a hydroxyl radicaldamaged guanine nucleotide that has been excised from DNA by endonuclease 75 repair [21]. Since repair occurs quickly, the excised DNA ends up in the saliva and urine which can then reflect the amount of DNA damage within the entire body [23].

High levels of $8-\mathrm{OHdG}$ have been detected in patients with: various malignancies, acute leukaemia, colorectal cancer [23], breast cancer [24], lung cancer [25], prostate cancers [21] and carcinogenesis in which oxidative DNA damage is caused by asbestos fibers [26]. Cancer diagnosis and survival rate depends heavily on early detection, and thus developing technologies applicable for sensitive and specific methods to detect cancer is a necessity. Early detection of cancer is difficult with traditional methods which do not have the selective sensitivity to detect biomarkers linked to specific cancers. Current manual laboratory-based observation diagnosis is labor intensive and can sometimes lead to error based upon interpretation of results. There is also the relatively high expense of ELISA testing kits together with the time delay to get a reliable and repeatable result.

8-OHdG therefore has a crucial role diagnosing the presence of various mutagenesis and can be used to diagnose cancer at as early stage. The objectives of this current research are to develop a single $370 \mathrm{~nm}$ width nanowire-based device, functionalized this using a durable chemical process which allows a reliable measurement to indicate the presence of a $2 \mu \mathrm{g} / \mathrm{ml}$ concentration in order to indicate the abnormal elevated presence $8-\mathrm{OHdG}$.

\section{RESEARCH METHODS}

\section{A. Fabrication of SiNW Immunosensors}

SiNW arrays were fabricated on a $10 \mathrm{~mm}^{2}$ silicon-oninsulator (SOI) substrates, which have a boron doped top Si layers with a thicknesses of $88 \mathrm{~nm}$. In order to obtain an ohmic contact to the SiNW, with consistent and repeatable $I-V$ (Current-voltage) characteristics, a higher doping concentration is necessary. SOI samples were first cleaned using a procedure consisting of solvent, acid and alkali cleaning stages which also incorporates a ten second hydrofluoric acid (HF) immersion stage after the acid and alkali cleansing stages. SiNWs were fabricated using a combination of electron beam lithography (EBL) using a Raith E-Line Instrument and optical lithography using a SUSS MicroTec Mask Aligner. SOI substrates were spin-coated with PMMA using a spin speed of $4000 \mathrm{rpm}$ for forty seconds to produce a $250 \mathrm{~nm}$ thick film. The PMMA was subsequently soft-baked at $85^{\circ} \mathrm{C}$ for two minutes before exposure to an electron beam for the directwrite EBL process.

The SiNW devices fabricated consisted of two microsized contact pads at either end of a SiNW. The PMMA was patterned using EBL parameters: aperture size of $30 \mathrm{~mm}$, acceleration voltage of $10 \mathrm{kV}$ and beam current of $0.201 \mathrm{nA}$. The micro-contact pads of the device were patterned using a dose area exposure of $100 \mathrm{~mA} / \mathrm{cm}^{2}$, and the SiNW channel was patterned using a line exposure dose of $500 \mathrm{pAs} / \mathrm{cm}^{2}$. The sample were developed in the PMMA developer isopropyl alcohol (IPA) methyl isobutylketone (MIBK) for one minute.

\section{B. Surface Functionalization}

Cyclic Voltammetry (CV) was used to electrochemically functionalize the surfaces of SiNWs with nitrobenzene. CV measurements were carried out at room temperature, with a potentiostat in a three-electrode configuration. All experiments used the SiNW chip array as the working electrode, a platinum (Pt) auxiliary electrode and an $\mathrm{Ag} / \mathrm{AgCl}$ electrode as the reference electrode. Initial attachment of nitro-phenyl groups to the SiNWs was performed using $\mathrm{CV}$ to induce an electrochemical reaction of 4-nitrobenzene diazonium tetrafluoroborate $(2 \mathrm{mM})$ in a non-aqueous acetonitrile per $0.1 \mathrm{M}$ tetrabutylammonium tetrafluoroborate $\left(\mathrm{NBu}_{4} \mathrm{BF}_{4}\right)$ electrolyte with the surface of the SiNW electrode, forming a thin film of covalently attached nitro-phenyl $\left(\mathrm{PhNO}_{2}\right)$ groups. $\mathrm{CV}$ was performed using a scan rate of $100 \mathrm{mV} / \mathrm{s}$. The CV process was repeated for twenty cycles to ensure the grafting reduction reaction is completed. After $\mathrm{PhNO}_{2}$ functionalization, the SiNW sample was cleaned with acetonitrile then rinsed with dichloromethane in order to remove any 

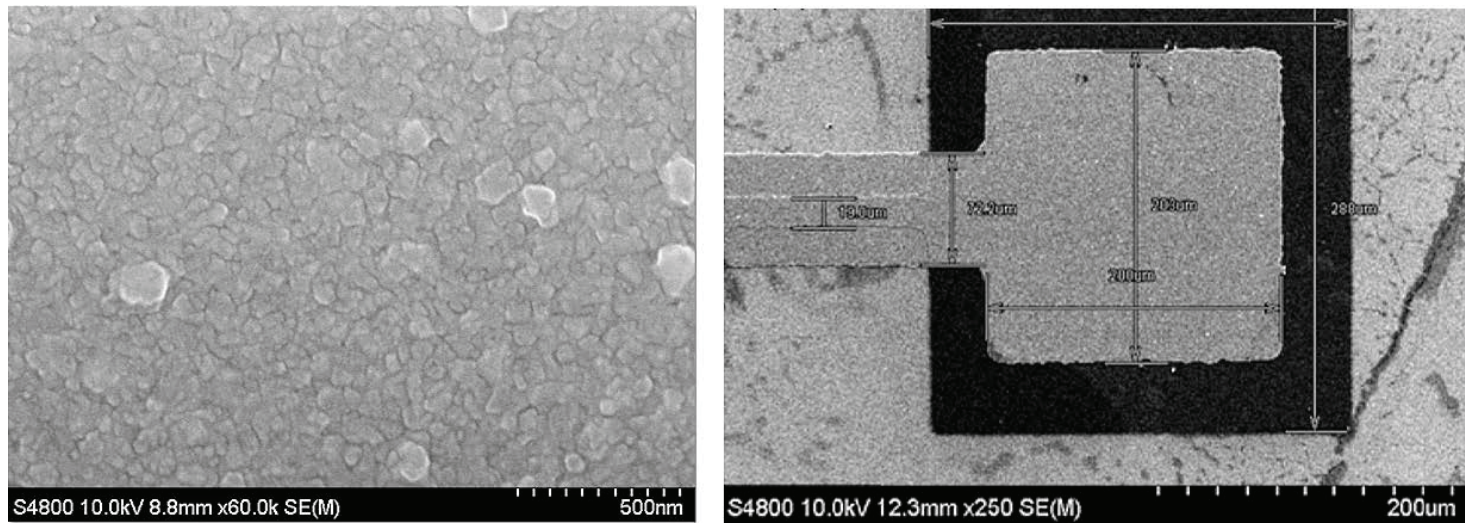

FIG. 2: Micrographs of the $300 \mathrm{~nm}$ thick aluminum contacts which were deposited onto the silicon surface.

physisorbed organic residues on the SiNW surface. The second step of the functionalization process is the reduction of the grafted nitrobenzene $\left(\mathrm{PhNO}_{2}\right)$ groups to aniline molecules $\left(\mathrm{PhNH}_{2}\right)$. The potential for reduction of the $\mathrm{PhNO}_{2}$ to an amine $\left(\mathrm{PhNH}_{2}\right)$ was identified using $\mathrm{CV}$ by sweeping through a potential range of $0 \mathrm{~V}$ to $+1 \mathrm{~V}$ in an $0.1 \mathrm{M} \mathrm{KCl}\left(\mathrm{H}_{2} \mathrm{O}: \mathrm{EtOH}, 90: 10\right)$ electrolyte with scan rate $100 \mathrm{mV} / \mathrm{s}$. The reduction of the $\mathrm{PhNO}_{2}$ to $\mathrm{PhNH}_{2}$ was completed using chronoamperometry with a constant voltage $(-0.9 \mathrm{~V})$ for $10 \mathrm{~min}$ using $\mathrm{Ag} / \mathrm{AgCl}$ as the reference electrode and $\mathrm{Pt}$ as an auxiliary electrode. To assess the progression of the reduction reaction towards completion, further CV measurements were performed at specific time intervals after chronoamperometry, in order to assess the progress of the reduction reaction toward completion. If the reaction was incomplete, chronoamperometry was performed for an additional 5 minutes using a subsequent CV scan to reassess the progress of the reaction. This process was repeated iteratively until the end point of the reduction is reached. This resulted in an aniline $\left(\mathrm{PhNH}_{2}\right)$ functionalized SiNW surface. The surface-bound amino group can subsequently be used to covalently bind to virtually any biomolecule containing a carboxyl group forming an amide link.

\section{Biofunctionalization}

Following chemical functionalization with the aniline linking molecule, the SiNW chip was biofunctionalized using an antibody bioreceptor, targeted against the oxidative stress biomarker, $8-\mathrm{OHdG}$. The primary antibody, mouse monoclonal anti- 8 hydroxyguanosine antibody (anti-8-OHdG, Abcam, UK), was diluted in phosphate buffered saline (PBS) $\mathrm{pH} 7.4$ to a concentration of $2 \mathrm{mg} / \mathrm{ml}$ and applied to the SiNW channels and incubated at $4^{\circ} \mathrm{C}$ for 4 hours before rinsing in deionised water. To confirm successful and specific binding of the primary antibodies to the SiNW channels, a secondary quantum dot labelled antibody, Qdot 655 goat $\mathrm{F}(\mathrm{ab}) 2$ anti-mouse IgG conjugate (Life Technologies Ltd, UK), was diluted to $20 \mathrm{nM}$ in PBS pH 7.4 and applied to the SiNW for 12 hours at $4^{\circ}$. The SiNW devices were then rinsed 5 times in deionized water to remove any excess unbound secondary antibodies, before drying. Fluorescence microscopy (LSCM) was used to verify successful antibody attachment to the SiNW surface via fluorescent emission from the conjugated secondary antibody. The excitation and emission wavelength of the Q-dot labeled secondary antibody were $530 \mathrm{~nm}$ and 651-658 nm respectively. Remaining free aniline groups on the SiNW surface were blocked using $5 \%$ bovine serum albumin (BSA) in PBS for 15 minutes at room temperature, to prevent nonspecific binding of the secondary antibody to any free surface amine groups. Fluorescence should then only be detected from the SiNW where the labeled secondary antibody has bound to the primary antibody.

\section{Microscopy and spectroscopy}

Scanning electron microscopy (SEM; Ultra-High Resolution FE-SEM S-4800, Hitachi) was carried out at $10 \mathrm{kV}$ acceleration voltage and a $9.8 \mathrm{~mA}$ emission current. The magnification was 2200 and working distance was $29.9 \mathrm{~mm}$. The SEM scan resolution was typically $640 \times 480$ pixels. Atomic force microscopy (AFM) was carried out using a JPK NanoWizards II AFM mounted on an inverted epifluorescence microscope (Zeiss Axiovert 200). Topographic images of SiNW were acquired in tapping mode in air, collected at a scan rate of $1.5 \mathrm{~Hz}$ over a scan area of $2 \mathrm{~mm}^{2}$. Laser scanning confocal microscopy (LSCM) was undertaken using an LSM 710 confocal microscope (Carl Zeiss Microscopy, Cambridge, UK). The LSCM scan resolution was typically $512 \times 512$ pixels with a pixel dwell time of $3.15 \mathrm{~ms}$. The laser excitation wavelength and optical light path filters were set appropriately for the fluorescent QD under examination (405 nm and 600-700 $\mathrm{nm}$ respectively).

\section{E. Electrical measurements}

Characterisation of SiNW channel device was performed using a Hewlett Packard 4142B DC parametric analyser running ITC characterization software in conjunction with a Karl Suss MP4 probe station. Currentvoltage $(I-V)$ measurements were performed using a voltage sweep of $-1.5 \mathrm{~V}$ to $+1.5 \mathrm{~V}$ between the two metal contacts of the SiNW device. 

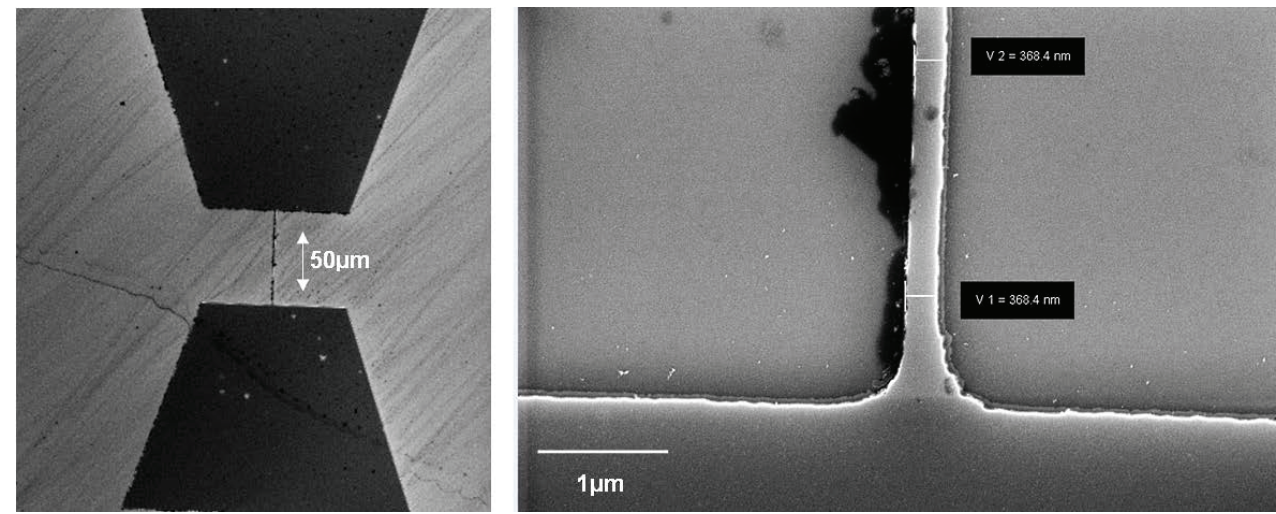

FIG. 3: Scanning electron micrograph of a $370 \mathrm{~nm}$ width $50 \mu \mathrm{m}$ length silicon nanowire which was produced using electron beam lithography.

\section{RESULTS}

\section{A. SiNW BioChip Fabrication}

Nano-based biosensing immunosensor approaches to bodily-fluid analysis provide the opportunity to isolate and then test for a sample of a biomarker within different bodily fluids using a single test. This configuration is particularly useful for analyzing cancer biomarkers, which can be present in different bodily fluids and provide a selective and accurate diagnosis. Scanning electron micrographs of the contacts are shown in Fig. 2, in which the surfaces roughness $\left(R_{z}\right)$ of this layer is sub $10 \mathrm{~nm}$. These contact pads were defined using photo lithography positive-tone resist. The sample was then thermally annealed using Rapid Thermal Annealing under a 50 sccm nitrogen flow in order to form an Ohmic contact.

A lift-off process, using a $100 \mathrm{~nm} \mathrm{Al}$ coating on top of the nanoscale PMMA pattern, was used to define an aluminum (Al) hard mask on top of the SOI substrate. The Al mask was used to selectively protect areas of silicon during the Reactive Ion Etching. Following etch removal of the exposed areas of the $200 \mathrm{~nm}$ silicon layer that was not protected by the $\mathrm{Al}$ mask, crystal line silicon nanowires are revealed. A second $1 \mathrm{~mm}$ thick $\mathrm{Al}$ deposition step was then performed to fabricate the metal contact pads at either end of the SiNW. This is achieved using an electron beam which has a nanoscale resolution to directly write the silicon to produce a $370 \mathrm{~nm}$ width SiNWs as shown in Fig. 3. The fabricated SiNW was then treated in an argon plasma in order to hydrogen terminate the silicon surface in readiness for subsequent chemical functionalization of the SiNW.

\section{B. Chemical Functionalization of SiNWs}

The electrochemical functionalization process is used to bind organic materials to the surface of a SiNW. The process examined within this research is binding antibodies targeted against $8 \mathrm{OHdG}$ to the surface of a nanowire as a means to detect the $8-\mathrm{OHdG}$ oxidative stress biomarker antibody which is linked to a range of cancers [27]. The phases of chemical and biological functionalization of a SiNW are shown schematically in Fig. 4.
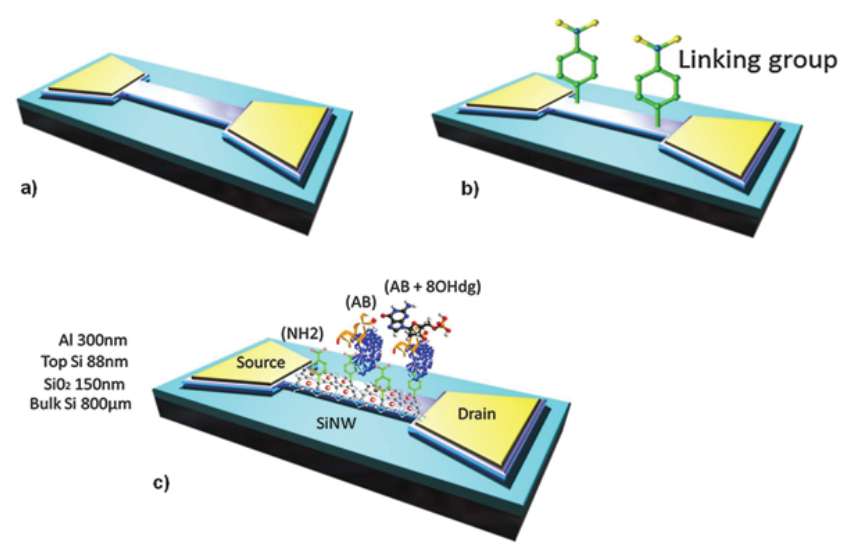

FIG. 4: Representation of a nanoscale SiNW-FET device with its surface biologically functionalized with antibodies targeted against 8-OHdG. (a) Fabricated SiNW (b) Attachment of Linking Groups using chemical functionalisation and (c) Attachment of antibodies targeted against $8-\mathrm{OHdG}$ and addition of 8-OHdG biomarker.

Exposure of the biomarker to the SiNW with attached 8-OHdG antibodies has decreased the resistance across the nanowire, in which there was a detectable linear response. As binding occurs, applying a negative gate voltage results in an accumulation of majority charge carrier positive holes and results in a corresponding increase in conductance. This indicates that the sensor is capable of detecting a target biomarker bonded to the surface of the nanowire. This process occurs within sixty-seconds of exposure to the target biomarker, making this a rapid cancer biomarker test.

As shown in Fig. 5 is the electrochemical process in which the silicon surface is chemically functionalized with linking groups. This process results in the surface modification at the molecular level of linkers that are bonded to the surface of each of the SiNWs.

Following the process of SiNW fabrication, the sample is cleaned with HF and then placed into an electrolyte in order to prevent any further reaction with the oxygen in the air. At this stage all the silicon atoms are fully coordinated and hydrogen terminated which leads to their enhanced stability in ambient environments. Unlike a normal surface which has unpassivated surface atoms, or dangling bonds the terminated surface can be handled for 


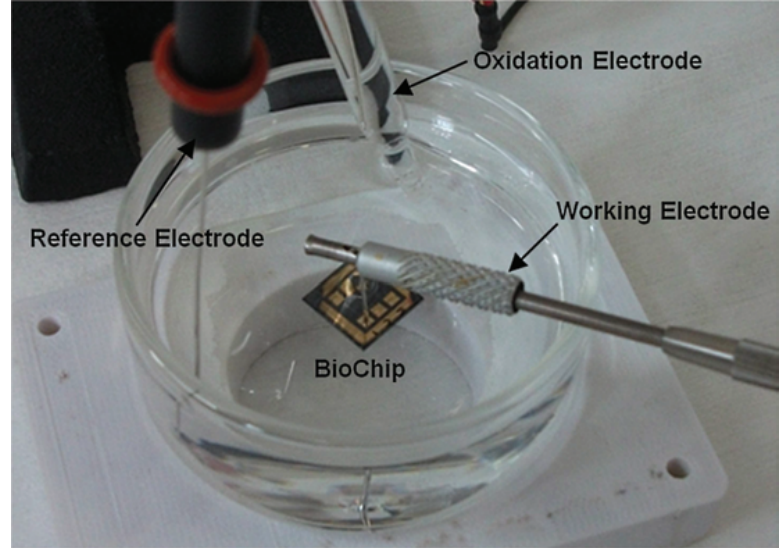

FIG. 5: Functionalization process in which the sample is first immersed in electrolyte following hydrogen-termination in hydrofluoric acid. The first stage results of attachment of diazonium salt to the silicon surface and second stage involves the reduction of $\mathrm{PhNO}_{2}$ to $\mathrm{PhNH}_{2}$.

several minutes in the air. The Hydrogen-silicon groups on the surface react with molecules that have terminal unsaturated bonds or diazo groups. Thus many different organic compounds with various functions can be introduced onto the silicon surface by the hydrosilylation of a Hydrogen-terminated surface. During this process the diazonium salt compound is used as an organic compound which shares a common functional group.

Following this, CV is used to electrochemically functionalize the surfaces of SiNWs with nitrobenzene. CV measurements were carried out with a potentiostat in a three-electrode configuration. All experiments used the SiNW chip array as the working electrode, a platinum auxiliary electrode and an $\mathrm{Ag} / \mathrm{AgCl}$ electrode as the reference electrode. This stage of the functionalization process is also performed at room temperature. The first functionalization step used an electrochemical reaction of 4-nitrobenzene diazonium tetrafluoroborate $(2 \mathrm{mM})$ in an acetonitrile / $0.1 \mathrm{M}$ tetrabutyl ammonium tetrafluoroborate $\left(\mathrm{NBu}_{4} \mathrm{BF}_{4}\right)$ electrolyte with the surface of the SiNW electrode, to form a thin film of covalently attached nitro phenyl $\left(\mathrm{PhNO}_{2}\right)$ groups on the SiNW surface. After the $\mathrm{PhNO}_{2}$ functionalization, the SiNW sample is cleaned with acetonitrile then rinsed with dichloromethane in order to remove any physisorbed organic residues on the SiNW surface.

Figure 6 shows the $\mathrm{CV}$ voltammogram taken in nonaqueous $\mathrm{Bu}_{4} \mathrm{NBF}_{4}$ in acetonitrile $0.1 \mathrm{mM}$ electrolyte, representing functionalization of the silicon surface with the nitro aryl diazonium salt (blue curve) which is shown by the first scan. After repeated voltammetry of twenty-five cycles the functionalization is complete and the silicon surface is saturated with the surface-attached nitrobenzene, which is shown by the final scan. The scan rate during this set of experiments was $100 \mathrm{mV} / \mathrm{s}$ in which no stirring of the solution was performed during the measurement of the $\mathrm{CV}$ as this can have an effect on the measurement taken. The first part of the scan ramps the voltage from $-0.9 \mathrm{~V}$ to $0 \mathrm{~V}$ and the changes observed indicate the start of the chemical functionalization process. Here an initial transfer of an electron from the electrode substrate to the aryl diazonium cation and results in the

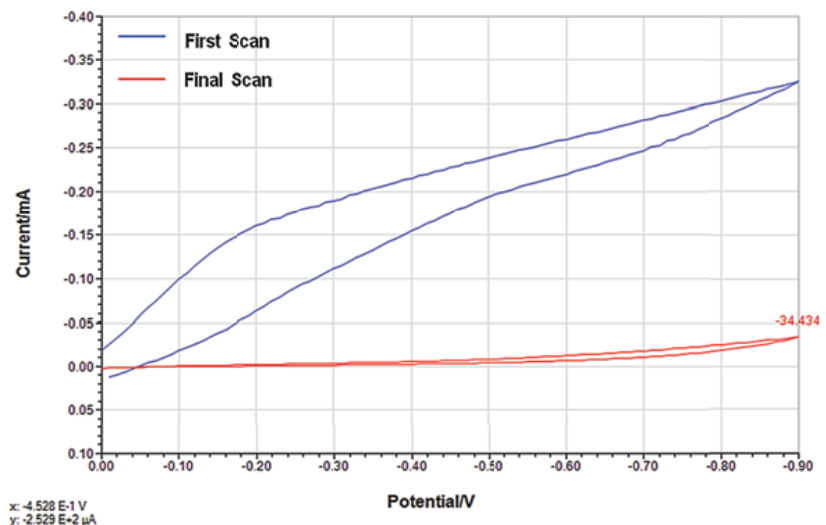

FIG. 6: Cyclic voltammogram of a chemically functionalized SiNW.

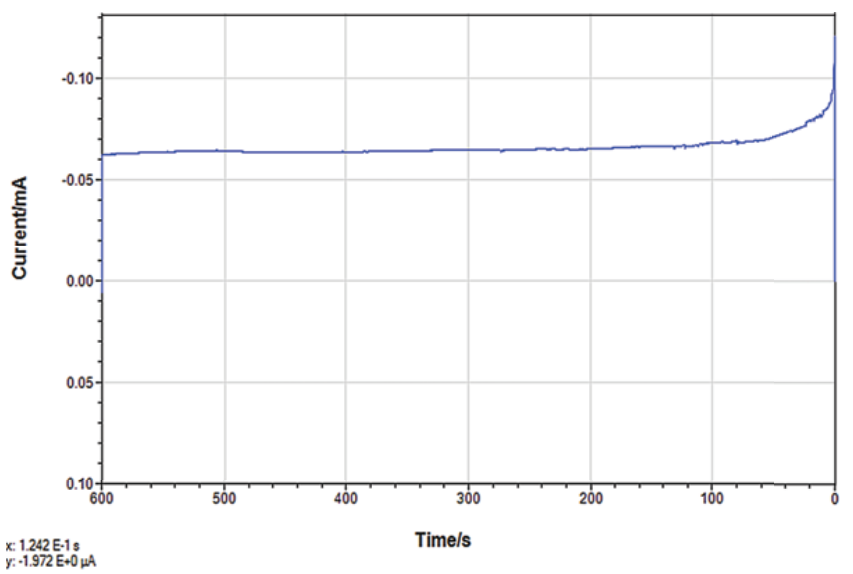

FIG. 7: Chronoamperometry of SiNW for the second stage of reduction. Chronoamperometry was measured in $0.1 \mathrm{M} \mathrm{KCl}$ in a solution of ethanol/water (1:9). Voltage applied was $-0.9 \mathrm{~V}$.

formation of an aryl radical through the release of a nitrogen molecule and subsequent reaction of this aryl radical with the silicon nanowire substrate occurs.

The attachment of the nitro-phenyl group to the silicon nanowire electron constitutes an overall reductive process. Because of the dissociation of the unstable diazoniumcation, to the aryl radical and nitrogen, this reduction reaction is an irreversible process. After the first reduction cycle, half a mono-layer of nitro-phenyl groups is attached to the SiNW surface, with $65 \%$ of all electrons are exchanged within the first cycle. As the reaction proceeds, the set of redox CV curves become closer together. This indicates that the reaction is nearing completion. The red curve shows the end point of the reaction, in which no more oxidation or reduction processes are occurring, which indicates that the reaction is completed.

The second stage of the chemical functionalization process reduced the $\mathrm{PhNO}_{2}$ to an amine $\left(\mathrm{NH}_{2}\right)$ in $0.1 \mathrm{M}$ $\mathrm{KCl}\left(\mathrm{H}_{2} \mathrm{O}: \mathrm{EtOH}, 90: 10\right)$ electrolyte. This resulted in an aniline $\left(\mathrm{PhNH}_{2}\right)$ functionalized SiNW surface being produced. The surface-bound amino group can then covalently bind to any biomolecule containing a carboxyl group forming an amide link. As shown in Fig. 7 is the chronoamperometry of SiNW, which is part of the second stage of reduction. Here surface nitrophenyls $\left(\mathrm{PhNO}_{2}\right)$ are converted to aminophenyls $\left(\mathrm{PhNH}_{2}\right)$ by electrochem- 


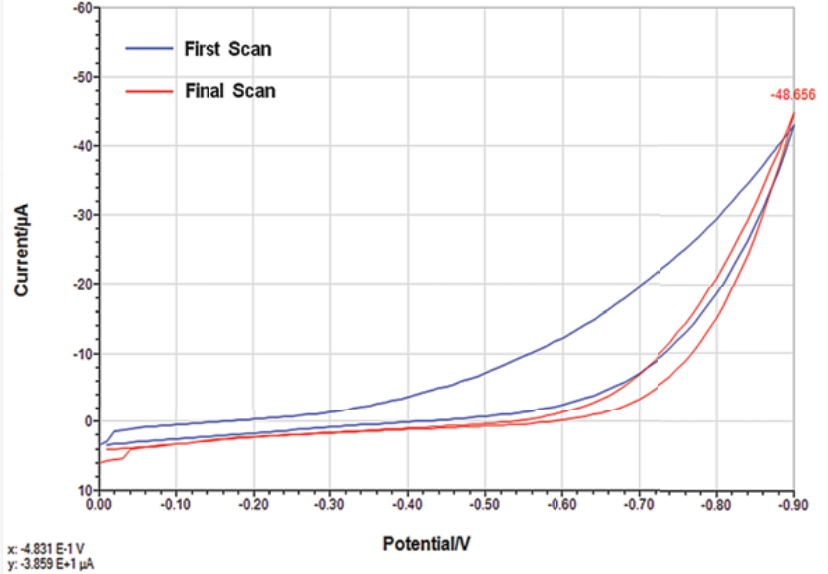

FIG. 8: Cyclic voltammetry of a SiNW when the nitro phenyl group is attached before and after applying chronoamperometry.

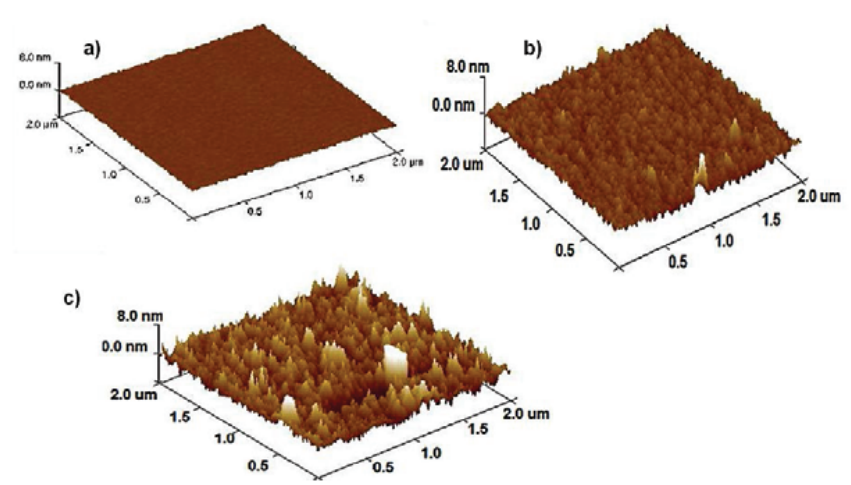

FIG. 9: AFM topography images of silicon surfaces (a) clean silicon $R_{a} 0.13 \mathrm{~nm}$, (b) after surface functionalization with $\mathrm{PhNO}_{2} / \mathrm{PhNH}_{2} R_{a} 0.96 \mathrm{~nm}$ and (c) after attachment with an antibody targeted against the 8-OHdG biomarker $R_{a} 2.3 \mathrm{~nm}$.

ical reduction. The electrode is subjected to two different electrochemical reduction strategies including Chronoamperometry at a constant potential and cyclic scanning between 0 and $-1 \mathrm{~V}$ versus $\mathrm{Ag} / \mathrm{AgCl}$ to find the best conditions for conversion. To identify the potential at which the $\mathrm{NO}_{2}$ to $\mathrm{NH}_{2}$ reduction occurs as shown in Fig. 8, a $\mathrm{CV}$ measurement is then used. The difference between the two CV curves is slight and shows amino-phenyl is attached to the SiNW surface. The cathodic sweep of the cyclic voltammogram are similar to each other.

Once the reduction voltage has been identified, chronoamperometry is used to complete the reduction reaction. In order to do this, chronoamperometry uses a reduction voltage identified from the $\mathrm{CV}$ measurement to achieve the $\mathrm{NO}_{2}$ to $\mathrm{NH}_{2}$ reduction. To assess the progression of the reduction reaction towards completion, further CV measurements are performed at specific time intervals. As the reduction reaction proceeds, the reduction and oxidation curves in the CV measurement move closer together. When the curves are superimposed on one another as shown in Fig. 8, this indicates the end point of the reduction reaction and completes the chemical functionalization of the silicon surface.

The bio-functionalized SiNW surfaces were investigated using AFM as shown in Fig. 9, in which acquisition

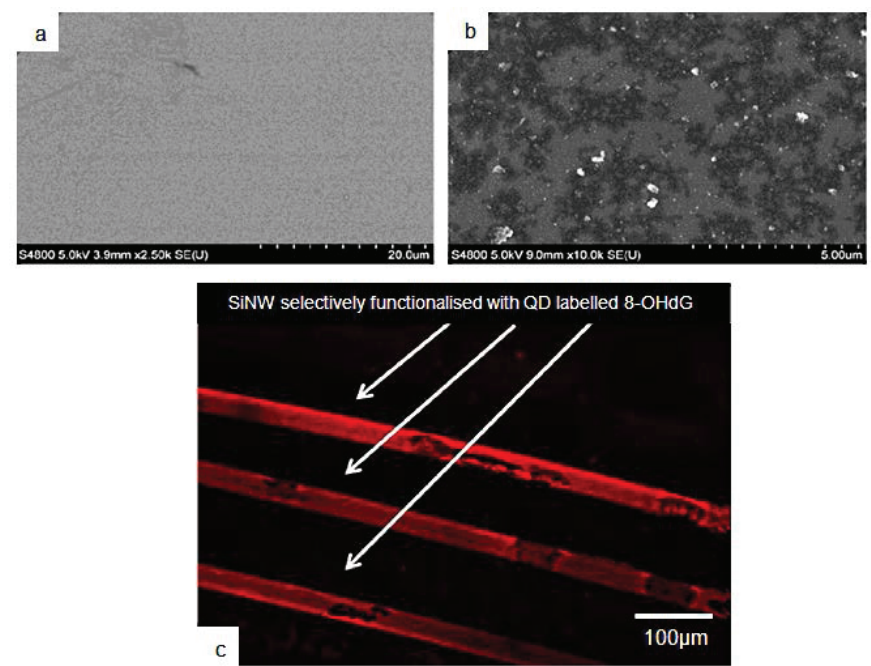

FIG. 10: SiNW (a) before functionalization (b) after functionalization and (c) laser scanning confocal micrograph of SiNWs selectively functionalized with a Quantum-dot labeled antibody targeted against anti $8-\mathrm{OHdG}$.

demonstrated clear differences in surface roughness following functionalization. Using this technique there is a difference in the morphology of the surface between each stage on the functionalization. In successive stages, the topography changed, from a flat surface to a rougher one.

When qualitatively comparing the surfaces, the sample with the antibody attached has a greater $R_{a}$ roughness of $2.3 \mathrm{~nm}$ compared with an $R_{a}$ of $0.13 \mathrm{~nm}$ for the blank surface. Thus attachment of the antibodies also appears to have modified the surface topography compared with amine groups only. This is because the antibodies are large macromolecules, and thus they produce a greater $R_{a}$ roughness as expected.

\section{Biofunctionalization and Detection of $8-\mathrm{OHdG}$ Biomarkers}

Following the chemical functionalization process with the aniline linking molecule, the SiNW chip was biofunctionalized using an antibody bioreceptor, targeted against the oxidative stress biomarker, $8-\mathrm{OHdG}$. The primary antibody was that of a mouse monoclonal anti- 8 hydroxyguanosine antibody which was diluted in PBS to a concentration of $2 \mathrm{mg} / \mathrm{ml}$ and applied to the SiNW and incubated at $4^{\circ} \mathrm{C}$ for 4 hours before rinsing in deionized water. In order to confirm the successful primary antibody attachment to the SiNW surface, this was achieved using fluorescent QD-labeled secondary antibody, which binds selectively to the surface of the primary antibodies.

As shown in Fig. 10(a) micrographs of the silicon before functionalization shows a clean surface without the presence of foreign species attached. Following functionalization, attachment of $\mathrm{PhNH}_{2}$ species to the silicon resulted in changing its topography as shown in Fig. 10(b). Following this, the detection of the QD-labeled antibody, attached to silicon surfaces, using fluorescence microscopy techniques enabled the success of the functionalization process to be evaluated. Strong, localized fluorescent signals can be seen from the SiNWs, with no non-specific 

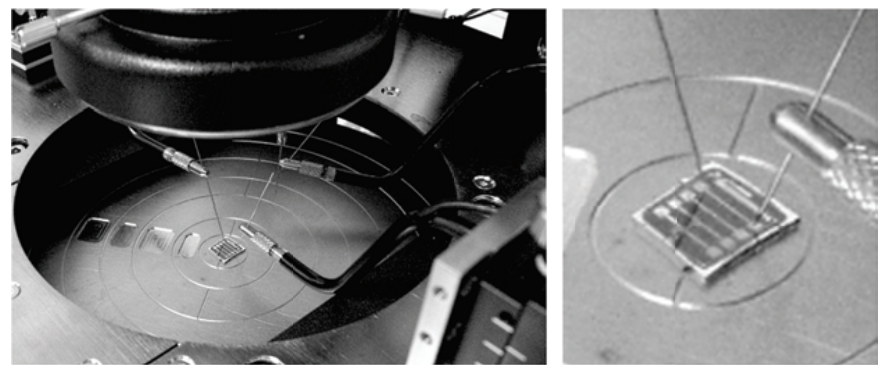

FIG. 11: Contact measurements taken directly from the biochip.

I(A)

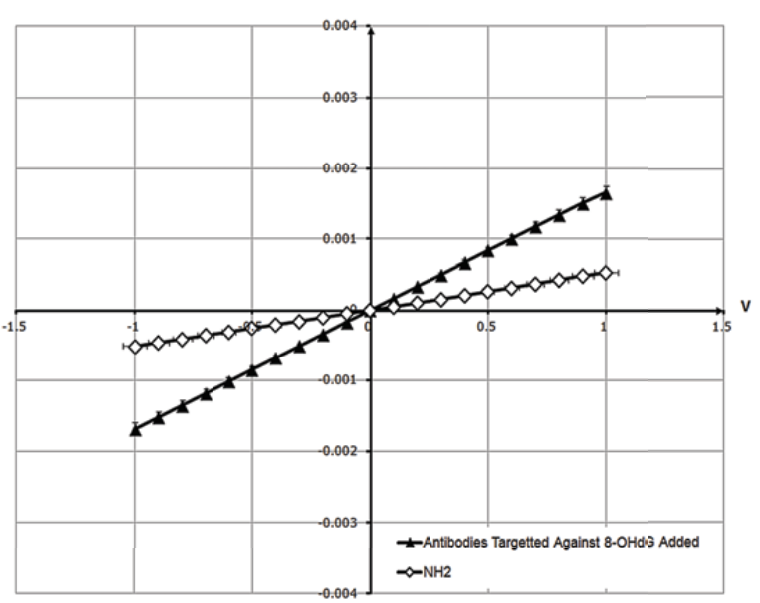

FIG. 12: Current/voltage characteristics of the chemically functionalized biochip and the addition of antibodies targeted against $8-\mathrm{OHdG}$.

labeling of the surrounding non-functionalized regions adjacent to the nanowires. The localized fluorescence signals indicate that the QDs have selectively bound to the primary antibodies which are attached only to the silicon surface.

Following this process, contact measurements were then taken directly from the biochip as shown in Fig. 11. During this process, changes in electrical resistance were measured during the biofunctionalization process in which the antibody targeted against 8 -OHdG is added to the surface.

Figure 12 shows the initial current/voltage characteristics of a SiNW after the electrochemical functionalization process. The current/voltage characterization of the sensor showed a change in resistance upon attachment of the antibody to SiNW surface. This shows that the antibodies are covalently attached to the surface via the linking group. As the antibody is applied to the surface then there is a decrease in resistance across the nanowire. This is because the $p$-type silicon used has the effect that as the hole current is increased by the negative gating potential. At this stage the biochip functionalization process is complete and it can be transferred into the device microfluidic packaging. Here the biomolecule carboxyl group of the antibodies binds with the linking group to form an amide link.

In order to test the control level of the biosensor, a $20 \mu \mathrm{L}$ sample of Phosphate Buffered Saline (PBS) solution was added. This is a water-based salt solution contain-
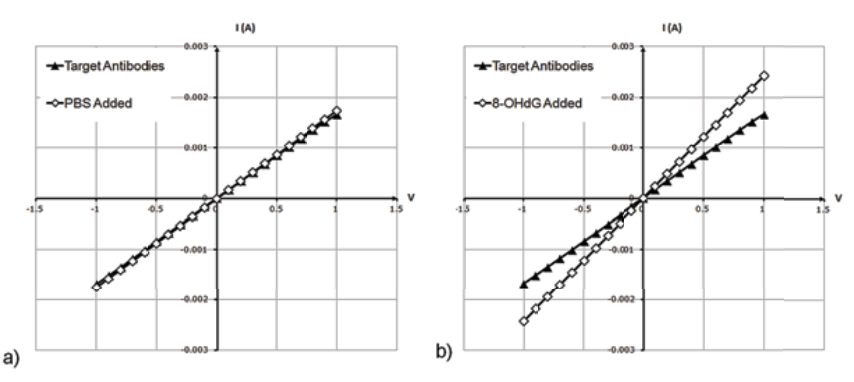

FIG. 13: Current/voltage characteristics of the SiNW (a) application of a buffer solution for comparison (b) application of the 8-OHdG biomarker.

ing sodium chloride and sodium phosphate. The buffer's phosphate groups help to maintain a constant $\mathrm{pH}$ and its osmolarity and ion concentrations of the solution match those of the human body. This control buffer solution is added to the SiNW to test the electrical conductance on an assay without the presence of a target biomarker to see if it causes a response. As Fig. 13(a) shows there is no difference in the conductance and this suggests that this is indeed the case that the buffer has no effect. This would be the case of a test was carried out on a person who did not have the presence of a target biomarker within a bodily fluid.

As shown in Fig. 13(b) when a $2 \mu \mathrm{g} / \mathrm{ml}$ sample of the $8 \mathrm{OHdG}$ target biomarker is added to the SiNW there is a significant decrease in the electrical resistance. When the surface modified device is exposed to the biomarker, it is the specific binding to the antibody receptor that leads to an increase in device conductance which also depends on the net charge of the biomolecule and the semiconductor. It is the real-time binding process that can be monitored in-situ with the change in conductance being logged which is of technical significance. Exposure of the biomarker to the SiNW with attached 8-OHdG antibodies has decreased the resistance across the nanowire, in which there was a detectable linear response. As binding occurs, applying a negative gate voltage results in an accumulation of majority charge carrier positive holes and results in a corresponding increase in conductance. This indicates that the sensor is capable of detecting a target biomarker bonded to the surface of the nanowire. This process occurs within sixty-seconds of exposure to the target biomarker, making this a rapid cancer biomarker test.

\section{DISCUSSION}

The introduction of point-of-care-based nanobiosensorbased systems in cancer diagnosis has arrived at an opportune time. With the increasing knowledge of cancer genomics and proteomics this has created further opportunities to detect biomarkers linked to various types of cancer. It is anticipated that nanowire-based technologies would be able to detect cancer at a very early stage through detecting biomarkers, providing a probability of treatment and subsequently cure [28]. In this research SiNWs have been successfully fabricated via a top-down fabrication approach using a combination of electron beam lithography and photolithography. 
This SiNW biosensor consists of a conductive silicon channel which is first chemically and subsequently biologically functionalized with an antibody targeted against 8-OHdG as shown in Fig. 1. This bioreceptor which is bound to the surface of the nanowire is able to detect the disease biomarker when it becomes attached to the surface as shown in Fig. 4. The gating effect results from changes in the surface charge density which induce a depletion or accumulation region in the SiNW consequently modifies the electrical conductance of the functionalized SiNW sensor. The detection limit is highly dependent on the diameter of the SiNW as shown in Fig. 3 with the greater sensitivity of smaller diameter nanowires, which is related to their higher surface to volume ratio. A key part of developing a biosensor technology to detect cancer biomarkers is the surface functionalization process. This was carried out by functionalization of SiNW with 8-OHdG bioreceptor antibodies in which this is achieved by firstly chemical functionalization of the surface with chemical linking groups as shown in Fig. 5. Here an electrochemical diazotization method for nitro phenyl attachment to surface was used and then a reduction process, converting the nitro group to an amine was carried out. This process is achieved within a short period of time and makes the functionalization process highly production efficient.

The process of grafting nitro-phenyl groups onto the SiNW surfaces was performed using cyclic voltammetry as shown in Fig. 6. The subsequent reduction of the grafted nitro-phenyl groups on the working electrode surface to amino-phenyl groups was performed using chronoamperometry as shown in Fig. 7 which applies a constant current to the working electrode. Cyclic voltammetry was also used to monitor the progress and extent of the functionalization and reduction reaction. As the $370 \mathrm{~nm}$ SiNWs used have a high surface-to-volume ratio they are selectively sensitive to surface events and can be used as an electrochemical biosensor.

Antibodies targeted against $8-\mathrm{OHdG}$ have been attached to SiNW and confocal fluorescence microscopy has been performed as shown in Fig. 10. Fluorescence from the labeled secondary antibodies was detected only from the region of the SiNWs, which indicates that the primary antibody is covalently bound to the SiNWs channel and not the silicon substrate. These results prove the concept of the specific and selective attachment of bioreceptor antibodies, which can subsequently be used for the detection of particular target biomolecules.

Changes in conductivity of the channel devices were observed at each stage of the functionalization process. The critical detection step; binding of the target 8OHdG biomarker to the SiNW-bound bioreceptor anti- body, yielded a detectable increase in the SiNW channel resistance as shown in Fig. 13. Elevated levels of 8-OHdG in saliva, serum and urine are associated with various cancers that are associated with oxidative stress damage mechanisms. The detection of cancer risk biomarker 8$\mathrm{OHdG}$ at a concentration of $2 \mu \mathrm{g} / \mathrm{ml}$ has been achieved. This concentration is useful as it is elevated enough to determine the presence of a high level of $8-\mathrm{OHdG}$. This suggests that this functionalized SiNW biosensors offers the appropriate level of sensitivity with the level of selective detection of the disease biomarker. This functionalization process can as a result be used to attach a wide range of bio-receptor molecules to the SiNW surface and then used as a sensor for early diagnosis and monitoring for a variety of different cancers. As $8-\mathrm{OHdG}$ is an oxidative stress biomarker which is related to the early existence of many different types of cancer, this key biomarker can be used to indicate the presence of oxidative DNA damage and act as a method for the early detection of potential mutagenesis.

\section{CONCLUSIONS}

Biomarkers detected by nano-based sensors offers a potentially powerful diagnostic method that has been further developed during this research. The key result of this research is that an integrated biosensor which can detect the presence of $8-\mathrm{OHdG}$ biomarkers has been developed. This device has been fabricated used a top down method for detecting the presence of this biomarker which is linked to cancer. The detection of elevated levels of this biomarker is critical in determining the early presence of various mutagenesis before initial symptoms appear. This durable surface chemical functionalization process developed during this research has involved the attachment of diazonium salt to the silicon nanowire with further stage which involved the reduction of $\mathrm{PhNO}_{2}$ to $\mathrm{PhNH}_{2}$. Subsequently an antibody targeted against $8-\mathrm{OHdG}$ was bound to the amine $\mathrm{NH}_{2}$ modified layer of the $370 \mathrm{~nm}$ width SiNW. During assessment through the use of current/voltage electrical probe monitoring a $2 \mu \mathrm{g} / \mathrm{ml}$ concentration of $8-\mathrm{OHdG}$ was detected. Comparisons with phosphate buffered saline solution added to the sensor was also observed to be negligible this ensures that the SiNW is functioning as an immunosensor. The functionalization process developed during this research can also be used to attach a wide range of bio-receptor molecules to the SiNW surface and monitor the presence for many different biomarker-based diseases.
[1] X. T. Vu, J. F. Eschermann, R. Stockmann, R. GhoshMoulick, A. Offenhäusser, and S. Ingebrandt, Phys. Status Solidi (a) 206, 426 (2009).

[2] F. Patolsky, B. P. Timko, G. Zheng, and C. M. Lieber, MRS Bull. 32, 142 (2007).

[3] F. Patolsky and C. M. Lieber, Mater. Today. 8, 20 (2005).

[4] F. Patolsky, B. P. Timko, G. Yu, Y. Fang, A. B. Greytak,
G. Zheng, and C. M. Lieber, Science 313, 1100 (2006).

[5] Y. Cui, Q. Wei, H. Park, and C. M. Lieber, Science 293, 1289 (2001).

[6] J. Wang, Analyst 130, 421 (2005).

[7] Y. Cui and C. M. Lieber, Science 291, 851 (2001).

[8] Y. Cui, Q. Wei, H. Park, and C. M. Lieber, Science 293, 1289 (2001). 
[9] Y. Cui, Z. Zhong, D. Wang, W. U. Wang, and C. M. Lieber, Nano Lett. 3, 149 (2003).

[10] G. Zheng, W. Lu, S. Jin, and C. M. Lieber, Adv. Mater. 16, 1890 (2004)

[11] J. Xiang, W. Lu, Y. Hu, Y. Wu, H. Yan, and C. M. Lieber, Nature 441, 489 (2006).

[12] S. M. Sze and K. K. Ng, Physics of Semiconductor Devices (Wiley, Hoboken, New Jersey, 2007), p. 293.

[13] G. Zheng, F. Patolsky, Y. Cui, W. U. Wang, and C. M. Lieber, Nat Biotechnol. 23, 1294 (2005).

[14] X. T. Vu, R. GhoshMoulick, J. F. Eschermann, R. Stockmann, A. Offenhäusser, and S. Ingebrandt, Sensors and Actuators B 144, 354 (2008).

[15] A. Gao, N. Lu, P. Dai, T. Li, H. Pei, X. Gao, Y. Gong, Y. Wang, and C. Fan, Nano Lett. 11, 3974 (2011).

[16] T. Kong, R. Su, B. Zhang, Q. Zhang, and G. Cheng, Biosensors and Bioelectronics 34, 267 (2012).

[17] Z. Li, B. Rajendran, T. I. Kamins, X. Li, Y. Chen, and R. S. Williams, Appl. Phys. A 80, 1257 (2005).

[18] Z. Gao, A. Agarwal, A. D. Trigg, N. Singh, C. Fang, C. Tung, Y. Fan, K. D. Buddharaju, and J. Kong, Anal. Chem. 79, 3291 (2007).

[19] S. W. Ryu, C. H. Kim, J. W. Han, C. J. Kim, C. Jung, H. G. Park, and Y. K. Choi, Biosensors and Bioelectronics
25, 2182 (2010).

[20] M. P. Stewart, F. Maya, D. V. Kosynkin, S. M. Dirk, J. J. Stapleton, C. L. McGuiness, D. L. Allara, and J. M. Tour, J. Am. Chem. Soc. 126, 370 (2004).

[21] L. L. Wu, C. Chiuan-Chian, C. Pi-Yueh, and T. W. James, Clinica Chimica Acta 339,133 (2004).

[22] J. Kuby, Immunology (W. H. Freeman and Company, New York, 1997).

[23] A. Valavanidis, T. Vlachogiannl, and C. Fiotakis, J. Environ. Sci. Health C 27, 120 (2009).

[24] A. Matsui, T. Ikeda, K. Enomoto, K. Hosoda, H. Nakashima, K. Omae, M. Watanabe, T. Hibi, and M. Kitajima, Cancer Lett. 151, 87 (2000).

[25] M. Erhola, S. Toyokuni, K. Okada, T. Tanaka, H. Hiai, H. Ochi, K. Uchida, T. Osawa, M. M. Nieminen, and H. Alho, FEBS Lett. 409, 287 (1997).

[26] S. Adachi, S. Yoshida, K. Kawamura, M. Takahashi, H. Uchida, Y. Odagiri, and K. Takemoto, Carcinogenesis 15, 753 (1994).

[27] R. Hirayama, Y. Furusawa, C. Murayama, Y. Kusano, and A. Ito, Radiation Phys. Chem. 78, 1207 (2009).

[28] J. Ludwig and J. Weinstein, Nat. Rev. Cancer 5, 845 (2005). 